\title{
Phosphine-Catalyzed [3+3] Domino Cycloaddition of Ynones and Azomethine Imines to Construct of Functionalized Hydropyridazine Derivatives
}

\author{
Ling Liang and You Huang*
}

\section{Table of contents}

General information .......................................................................................................

General procedure for [3+3] cycloaddition reaction.........................................s1

Analytical data for all new compounds ..............................................................s1

NMR spectra for all new compounds ................................................................ S8

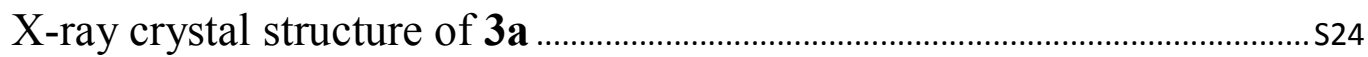




\section{General information}

All the solvents were used without further purification. ${ }^{1} \mathrm{H}$ NMR (400 MHz) and ${ }^{13} \mathrm{C}$ NMR (100 $\mathrm{MHz}$ ) were recorded on a Bruker $\mathrm{AV} 400(400 \mathrm{MHz})$ spectrometer with $\mathrm{CDCl}_{3}$ as solvent. Chemical shifts were recorded in parts per million (ppm) relative to tetramethylsilane as an internal reference. Coupling constants are given in Hz. The following abbreviations are used to indicate the multiplicity: s, singlet; $\mathrm{d}$, doublet: $\mathrm{t}$, triplet; $\mathrm{q}$, quartet; dd, doublet of doublet; $\mathrm{m}$, multiplet; HRMS were obtained on an VG ZAB-HS mass spectrometer with ESI resource. Melting points were measured on a RY-I apparatus and are reported uncorrected. IR spectra were recorded as $\mathrm{KBr}$ disks on a Nicolet 380 FT-IR spectrometer. Column chromatography was performed on silica gel 200-300 mesh. Ynones ${ }^{1}$ and azomethine imines ${ }^{2}$ compound were prepared according to the known methods

1 (a) Schuler, M.; Silva, F.; Bobbio, C.; Tessier, A.; Gouvemeur, V. Angew. Chem. Int. Ed., 2008, 47, 7927-7930. (b) Chan, K-H.; Leong, W-K.; Jaouen, G.; Lecelerq, L.; Top, S.; Vessieres, A. J. Organomet. Chem. 2006, 691, 9-19;

2 (a) Shintani, R.; Fu, G. C. J. Am. Chem. Soc. 2003, 125, 10778-; (b) Shintani, R.; Hayashi, T. J. Am. Chem. Soc. 2006, 128, 6330-6331. (c) Xu, X.; Qian, Y.; Zavalij, P. Y.; Doyle, M. P. J. Am. Chem. Soc. 2013, 135, 1244-1247.

\section{General procedure for $[3+3]$ cycloaddition reaction}

Ynones $1(0.45 \mathrm{mmol})$ and azomethine imines $2(0.3 \mathrm{mmol})$ were dissolved in $5.0 \mathrm{~mL}$ solvent $\left(n-\mathrm{BuOH}+\mathrm{CHCl}_{3}\right)$, and then $\mathrm{PPh}_{3}(30 \mathrm{~mol} \%)$ was added to this solution. The reaction was stirred at $30{ }^{\circ} \mathrm{C}$. As indicated by TCL, after completed conversion, all volatiles were removed in vacuo and the residue was purified via column chromatography (Petroleum ether/ethyl acetate $=4: 1$ ).

\section{Analytical data for all new compounds}

\section{(Z)-8-benzylidene-5-(4-bromophenyl)tetrahydro-1H-pyrazolo[1,2-a]pyridazine-1, 7(8H)-dione (3a)}

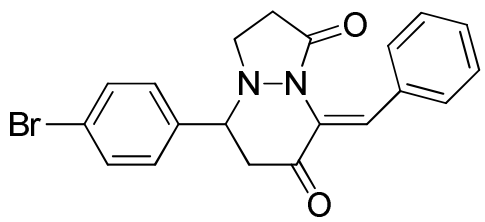

yellow solid (97 $\mathrm{mg}, 82 \%$ yield); $\mathrm{mp} .:$ 167-169 ${ }^{\circ} \mathrm{C}$ 
${ }^{1} \mathrm{H}$ NMR $\left(400 \mathrm{MHz}, \mathrm{CDCl}_{3}\right) \delta 7.59(\mathrm{~d}, J=8.3 \mathrm{~Hz}, 2 \mathrm{H}), 7.54(\mathrm{~d}, J=6.1 \mathrm{~Hz}, 2 \mathrm{H}), 7.50(\mathrm{~s}, 1 \mathrm{H}), 7.43-$ $7.36(\mathrm{~m}, 3 \mathrm{H}), 7.31(\mathrm{~s}, 1 \mathrm{H}), 7.29(\mathrm{~s}, 1 \mathrm{H}), 4.35(\mathrm{dd}, J=10.1,4.9 \mathrm{~Hz}, 1 \mathrm{H}), 3.58(\mathrm{td}, J=12.4,8.6 \mathrm{~Hz}, 1 \mathrm{H})$, $3.29-3.17(\mathrm{~m}, 1 \mathrm{H}), 2.96(\mathrm{dd}, J=18.5,4.8 \mathrm{~Hz}, 1 \mathrm{H}), 2.91-2.75(\mathrm{~m}, 2 \mathrm{H}), 2.52-2.38(\mathrm{~m}, 1 \mathrm{H})$.

${ }^{13} \mathrm{C}$ NMR $\left(100 \mathrm{MHz} \mathrm{CDCl}_{3}\right) \delta 191.10,169.87,138.47,133.60,132.58,130.86,130.06,128.97,128.49$, $128.32,128.13,122.79,65.14,45.91,45.66,28.62$.

IR (KBr): 2317.52, 1716.43, 1696.89, 1605.85, 1489.18, 1393.49, 1173.98, 1011.16, 834.64, 692.41 $\mathrm{cm}^{-1}$

HRMS (ESI): m/z calcd for $\mathrm{C}_{20} \mathrm{H}_{18} \mathrm{BrN}_{2} \mathrm{O}_{2},[\mathrm{M}+\mathrm{H}]^{+}, 397.0552$; found: 397.0560 .

\section{(Z)-8-benzylidene-5-(4-chlorophenyl)tetrahydro-1H-pyrazolo[1,2-a]pyridazine-1, 7(8H)-dione (3b)}

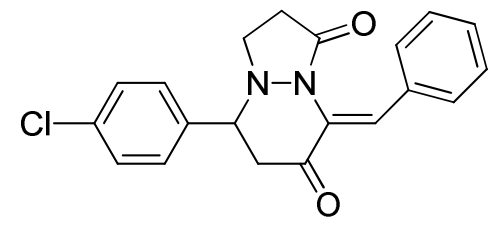

yellow solid (86 mg, $81 \%$ yield); mp.: $169-171^{\circ} \mathrm{C}$

${ }^{1} \mathrm{H}$ NMR $\left(400 \mathrm{MHz}, \mathrm{CDCl}_{3}\right) \delta 7.51(\mathrm{~d}, J=6.4 \mathrm{~Hz}, 2 \mathrm{H}), 7.48(\mathrm{~s}, 1 \mathrm{H}), 7.41(\mathrm{~d}, J=8.1 \mathrm{~Hz}, 2 \mathrm{H}), 7.38-$

$7.30(\mathrm{~m}, 5 \mathrm{H}), 4.33(\mathrm{dd}, J=9.7,4.7 \mathrm{~Hz}, 1 \mathrm{H}), 3.55(\mathrm{dd}, J=20.7,12.2 \mathrm{~Hz}, 1 \mathrm{H}), 3.26-3.15(\mathrm{~m}, 1 \mathrm{H}), 2.93$ $(\mathrm{dd}, J=17.5,3.1 \mathrm{~Hz}, 1 \mathrm{H}), 2.88-2.76(\mathrm{~m}, 2 \mathrm{H}), 2.48-2.36(\mathrm{~m}, 1 \mathrm{H})$.

${ }^{13} \mathrm{C}$ NMR $\left(100 \mathrm{MHz}, \mathrm{CDCl}_{3}\right) \delta 191.13,169.86,137.93,134.71,133.59,130.85,130.06,129.64,128.65$, $128.49,128.37,128.11,65.13,45.96,45.64,28.63$.

IR (KBr): 1717.38, 1696.64, 16006.50, 1491.03, 1173.03, ,1098.36, 1015.28, 837.09, $691.90 \mathrm{~cm}^{-1}$ HRMS (ESI): m/z calcd for $\mathrm{C}_{20} \mathrm{H}_{18} \mathrm{ClN}_{2} \mathrm{O}_{2},[\mathrm{M}+\mathrm{H}]^{+}, 353.1057$; found: 353.1061 .

\section{(Z)-8-benzylidene-5-(3-chlorophenyl)tetrahydro-1H-pyrazolo[1,2-a]pyridazine-1,} 7(8H)-dione (3c)

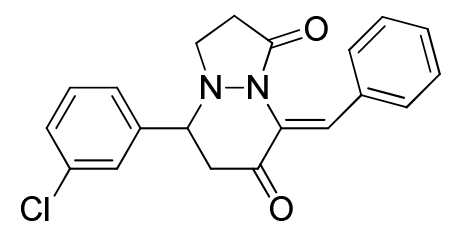

yellow solid ( $81 \mathrm{mg}, 76 \%$ yield); mp.: $183-185^{\circ} \mathrm{C}$

${ }^{1} \mathrm{H}$ NMR (400 MHz, $\left.\mathrm{CDCl}_{3}\right) \delta 7.54-7.49$ (m, 2H), 7.47 (s, 1H), 7.40 (s, 1H), 7.36 (dd, J= 7.3, 5.2 Hz, $5 \mathrm{H}), 7.30-7.24(\mathrm{~m}, 1 \mathrm{H}), 4.33(\mathrm{dd}, J=10.1,5.0 \mathrm{~Hz}, 1 \mathrm{H}), 3.57(\mathrm{td}, J=12.5,8.5 \mathrm{~Hz}, 1 \mathrm{H}), 3.27-3.17$

$(\mathrm{m}, 1 \mathrm{H}), 2.94(\mathrm{dd}, J=18.5,5.0 \mathrm{~Hz}, 1 \mathrm{H}), 2.88-2.77(\mathrm{~m}, 2 \mathrm{H}), 2.44(\mathrm{dd}, J=17.1,7.7 \mathrm{~Hz}, 1 \mathrm{H})$.

${ }^{13} \mathrm{C}$ NMR $\left(100 \mathrm{MHz}, \mathrm{CDCl}_{3}\right) \delta 190.96,169.85,141.48,135.27,133.56,130.85,130.75,130.08,129.12$, $128.50,128.45,128.06,127.43,125.41,65.27,45.90,45.76,28.61$.

IR (KBr): 3059.16, 1714.79, 1605.54, 1393.44, 1197.63, 1174.93, 754.27, $694.37 \mathrm{~cm}^{-1}$

HRMS (ESI): $\mathrm{m} / \mathrm{z}$ calcd for $\mathrm{C}_{20} \mathrm{H}_{18} \mathrm{ClN}_{2} \mathrm{O}_{2},[\mathrm{M}+\mathrm{H}]^{+}, 353.1057$; found: 353.1059 .

(Z)-8-benzylidene-5-(2-chlorophenyl)tetrahydro-1H-pyrazolo[1,2-a]pyridazine-1, 7(8H)-dione (3d) 


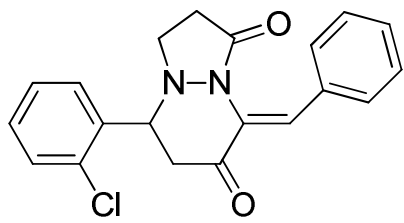

yellow solid ( $85 \mathrm{mg}, 81 \%$ yield); mp.: $226-228^{\circ} \mathrm{C}$

${ }^{1} \mathrm{H}$ NMR $\left(400 \mathrm{MHz}, \mathrm{CDCl}_{3}\right) \delta 7.59$ (d, $\left.J=7.4 \mathrm{~Hz}, 1 \mathrm{H}\right), 7.53(\mathrm{~d}, J=6.3 \mathrm{~Hz}, 2 \mathrm{H}), 7.49$ (s, $\left.1 \mathrm{H}\right), 7.43$ (d, $J=7.9 \mathrm{~Hz}, 1 \mathrm{H}), 7.40-7.33(\mathrm{~m}, 4 \mathrm{H}), 7.33-7.29(\mathrm{~m}, 1 \mathrm{H}), 5.01(\mathrm{dd}, J=10.4,4.8 \mathrm{~Hz}, 1 \mathrm{H}), 3.57(\mathrm{td}, J=$ $12.5,8.5 \mathrm{~Hz}, 1 \mathrm{H}), 3.23-3.13(\mathrm{~m}, 1 \mathrm{H}), 3.01(\mathrm{dd}, J=18.6,4.8 \mathrm{~Hz}, 1 \mathrm{H}), 2.96-2.88(\mathrm{~m}, 1 \mathrm{H}), 2.79(\mathrm{dd}, J$

$=18.6,10.4 \mathrm{~Hz}, 1 \mathrm{H}), 2.46(\mathrm{dd}, J=17.5,8.0 \mathrm{~Hz}, 1 \mathrm{H})$.

${ }^{13} \mathrm{C}$ NMR $\left(100 \mathrm{MHz}, \mathrm{CDCl}_{3}\right) \delta 191.09,170.31,136.88,133.59,133.43,130.90,130.09,129.97,129.66$, $128.51,128.25,128.16,60.77,45.98,44.47,29.06$.

IR (KBr): 2317.93, 1718.16, 1697.33, 1607.26, 1394.16, 1236.15, 1195.82, 1173.12, 753.59, 690.89 $\mathrm{cm}^{-1}$

HRMS (ESI): m/z calcd for $\mathrm{C}_{20} \mathrm{H}_{18} \mathrm{ClN}_{2} \mathrm{O}_{2},[\mathrm{M}+\mathrm{H}]^{+}, 353.1057$; found: 353.1057 .

\section{(Z)-8-benzylidene-5-(4-fluorophenyl)tetrahydro-1H-pyrazolo[1,2-a]pyridazine-1, 7(8H)-dione (3e)}<smiles>O=C1CC(c2ccc(F)cc2)N2CCC(=O)N2/C1=C\c1ccccc1</smiles>

yellow solid (69 mg, 68\% yield); mp.: $191-193{ }^{\circ} \mathrm{C}$

${ }^{1} \mathrm{H}$ NMR (400 MHz, $\left.\mathrm{CDCl}_{3}\right) \delta 7.51(\mathrm{~d}, J=6.3 \mathrm{~Hz}, 2 \mathrm{H}), 7.47(\mathrm{~s}, 1 \mathrm{H}), 7.40-7.32(\mathrm{~m}, 5 \mathrm{H}), 7.12(\mathrm{t}, J=$ $8.3 \mathrm{~Hz}, 2 \mathrm{H}), 4.34$ (dd, $J=9.6,4.9 \mathrm{~Hz}, 1 \mathrm{H}), 3.53(\mathrm{dd}, J=20.7,12.2 \mathrm{~Hz}, 1 \mathrm{H}), 3.30-3.14$ (m, 1H), 2.93 (dd, 1H), $2.89-2.76(\mathrm{~m}, 2 \mathrm{H}), 2.42(\mathrm{~s}, 1 \mathrm{H})$.

${ }^{13} \mathrm{C}$ NMR $\left(100 \mathrm{MHz} \mathrm{CDCl}_{3}\right) \delta 191.33,169.92,164.01,161.54,135.29,133.62,130.85,130.03,129.08$, $129.00,128.48,128.27,128.16,116.49,116.28,65.03,46.13,45.52,28.65$.

IR (KBr): 1715.77, 1696.32, 1605.26, 1509.61, 1393.10, 1227.12, 1174.18, 842.57, 691.75, 599.47 $\mathrm{cm}^{-1}$

HRMS (ESI): $\mathrm{m} / \mathrm{z}$ calcd for $\mathrm{C}_{20} \mathrm{H}_{18} \mathrm{FN}_{2} \mathrm{O}_{2},[\mathrm{M}+\mathrm{H}]^{+}, 337.1352$; found: 337.1347 .

\section{(Z)-8-benzylidene-5-phenyltetrahydro-1H-pyrazolo[1,2-a]pyridazine-1,7(8H)-dio} ne (3f)<smiles>O=C1CC(c2ccccc2)N2CCC(=O)N2/C1=C\c1ccccc1</smiles>

yellow solid (62 mg, $65 \%$ yield); mp.: $181-183{ }^{\circ} \mathrm{C}$

${ }^{1} \mathrm{H}$ NMR (400 MHz, $\left.\mathrm{CDCl}_{3}\right) \delta 7.52(\mathrm{~d}, J=6.6 \mathrm{~Hz}, 2 \mathrm{H}), 7.48(\mathrm{~s}, 1 \mathrm{H}), 7.45-7.41(\mathrm{~m}, 2 \mathrm{H}), 7.39-7.36$ $(\mathrm{m}, 4 \mathrm{H}), 7.35-7.31(\mathrm{~m}, 2 \mathrm{H}), 4.42-4.29(\mathrm{~m}, 1 \mathrm{H}), 3.54(\mathrm{dd}, J=21.0,12.4 \mathrm{~Hz}, 1 \mathrm{H}), 3.36-3.19(\mathrm{~m}$, $1 \mathrm{H}), 3.06-2.87(\mathrm{~m}, 2 \mathrm{H}), 2.81(\mathrm{~s}, 1 \mathrm{H}), 2.41(\mathrm{~s}, 1 \mathrm{H})$. 
${ }^{13} \mathrm{C}$ NMR $\left(100 \mathrm{MHz}, \mathrm{CDCl}_{3}\right) \delta 191.58,170.09,139.34,133.69,130.85,129.97,129.40,128.92,128.46$, 128.26, 128.17, 127.34, 65.88, 46.06, 45.59, 28.74.

IR (KBr): 1716.27, 1696.09, 1605.50, 1393.61, 1235.78, 1174.01, 1023.72, 755.57, $696.04 \mathrm{~cm}^{-1}$ HRMS (ESI): $\mathrm{m} / \mathrm{z}$ calcd for $\mathrm{C}_{20} \mathrm{H}_{19} \mathrm{~N}_{2} \mathrm{O}_{2}$, [M+H] $]^{+}, 319.1447$; found: 319.1452 .

(Z)-8-benzylidene-5-(p-tolyl)tetrahydro-1H-pyrazolo[1,2-a]pyridazine-1,7(8H)-di one (3g)

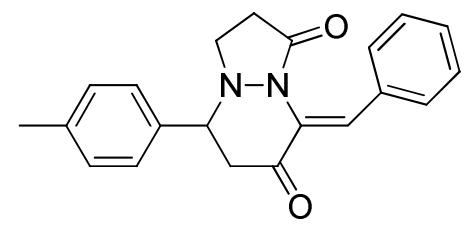

yellow solid (60 $\mathrm{mg}, 60 \%$ yield); $\mathrm{mp} .: 153-155^{\circ} \mathrm{C}$

${ }^{1} \mathrm{H}$ NMR (400 MHz, $\left.\mathrm{CDCl}_{3}\right) \delta 7.52(\mathrm{~d}, J=1.7 \mathrm{~Hz}, 1 \mathrm{H}), 7.51(\mathrm{~s}, 1 \mathrm{H}), 7.47(\mathrm{~s}, 1 \mathrm{H}), 7.39-7.30(\mathrm{~m}, 3 \mathrm{H})$, $7.28-7.25(\mathrm{~m}, 1 \mathrm{H}), 7.24(\mathrm{~d}, J=4.6 \mathrm{~Hz}, 3 \mathrm{H}), 4.42-4.23(\mathrm{~m}, 1 \mathrm{H}), 3.51(\mathrm{td}, J=12.3,8.5 \mathrm{~Hz}, 1 \mathrm{H}), 3.30$ $-3.17(\mathrm{~m}, 1 \mathrm{H}), 3.01-2.86(\mathrm{~m}, 2 \mathrm{H}), 2.80(\mathrm{~s}, 1 \mathrm{H}), 2.47-2.29(\mathrm{~m}, 4 \mathrm{H})$.

${ }^{13} \mathrm{C} \mathrm{NMR}\left(100 \mathrm{MHz}, \mathrm{CDCl}_{3}\right) \delta 191.79,170.11,138.81,136.30,133.74,130.85,130.01,129.92,128.44$, 128.32, 128.03, 127.28, 65.58, 46.09, 45.49, 28.75, 21.24 .

IR (KBr): $1717.33,1696.25,1606.36,1393.47,1253.31,1173.80,819.91,757.08,692.13 \mathrm{~cm}^{-1}$ HRMS (ESI): $\mathrm{m} / \mathrm{z}$ calcd for $\mathrm{C}_{21} \mathrm{H}_{21} \mathrm{~N}_{2} \mathrm{O}_{2}$, [M+H] $]^{+}, 333.1603$; found: 333.1610 .

(Z)-8-benzylidene-5-(4-methoxyphenyl)tetrahydro-1H-pyrazolo[1,2-a]pyridazine $-1,7(8 \mathrm{H})$-dione (3h)

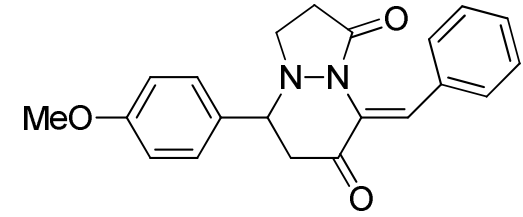

yellow solid (40 mg, $38 \%$ yield); $\mathrm{mp} .: 156-158{ }^{\circ} \mathrm{C}$

${ }^{1} \mathrm{H}$ NMR $\left(400 \mathrm{MHz}, \mathrm{CDCl}_{3}\right) \delta 7.54-7.50(\mathrm{~m}, 2 \mathrm{H}), 7.47(\mathrm{~s}, 1 \mathrm{H}), 7.40-7.32(\mathrm{~m}, 3 \mathrm{H}), 7.29(\mathrm{~d}, J=8.5$ $\mathrm{Hz}, 2 \mathrm{H}), 6.94(\mathrm{~d}, J=8.7 \mathrm{~Hz}, 2 \mathrm{H}), 4.29(\mathrm{dd}, 1 \mathrm{H}), 3.83(\mathrm{~s}, 3 \mathrm{H}), 3.50(\mathrm{td}, J=12.3,8.5 \mathrm{~Hz}, 1 \mathrm{H}), 3.30-$ $3.21(\mathrm{~m}, 1 \mathrm{H}), 2.97-2.87(\mathrm{~m}, 2 \mathrm{H}), 2.80(\mathrm{~s}, 1 \mathrm{H}), 2.35(\mathrm{~s}, 1 \mathrm{H})$.

${ }^{13} \mathrm{C}$ NMR $\left(100 \mathrm{MHz}, \mathrm{CDCl}_{3}\right) \delta 191.84,170.07,159.94,133.72,130.83,129.93,128.59,128.44,128.28$, 128.06, 114.67, 65.25, 55.38, 46.11, 45.38, 28.76 .

IR (KBr): 1715.78, 1695.70, 1608.14, 1512.97, 1247.61, 1174.09, 1208.64, 838.15, $692.41 \mathrm{~cm}^{-1}$ HRMS (ESI): $\mathrm{m} / \mathrm{z}$ calcd for $\mathrm{C}_{21} \mathrm{H}_{21} \mathrm{~N}_{2} \mathrm{O}_{3}$, [M+H] $]^{+}, 349.1552$; found: 349.1552 .

(Z)-8-benzylidene-5-(4-nitrophenyl)tetrahydro-1H-pyrazolo[1,2-a]pyridazine-1,7 (8H)-dione (3i)<smiles>O=C1CC(c2ccc([N+](=O)[O-])cc2)N2CCC(=O)N2/C1=C\c1ccccc1</smiles> 
yellow solid (52 mg, $48 \%$ yield); $\mathrm{mp} .: 156-158{ }^{\circ} \mathrm{C}$

${ }^{1} \mathrm{H}$ NMR $\left(400 \mathrm{MHz}, \mathrm{CDCl}_{3}\right) \delta 8.31(\mathrm{~d}, J=8.7 \mathrm{~Hz}, 2 \mathrm{H}), 7.60(\mathrm{~d}, J=8.7 \mathrm{~Hz}, 2 \mathrm{H}), 7.51(\mathrm{dd}, J=7.2,5.7$

$\mathrm{Hz}, 3 \mathrm{H}), 7.42-7.32$ (m, 3H), 4.51 (dd, $J=10.3,4.9 \mathrm{~Hz}, 1 \mathrm{H}), 3.67-3.56(\mathrm{~m}, 1 \mathrm{H}), 3.22-3.10(\mathrm{~m}, 1 \mathrm{H})$, $2.97(\mathrm{dd}, J=18.5,4.9 \mathrm{~Hz}, 1 \mathrm{H}), 2.91-2.73(\mathrm{~m}, 2 \mathrm{H}), 2.48(\mathrm{dd}, J=17.4,8.1 \mathrm{~Hz}, 1 \mathrm{H})$.

${ }^{13} \mathrm{C}$ NMR $\left(100 \mathrm{MHz}, \mathrm{CDCl}_{3}\right) \delta 190.26,169.63,148.07,146.59,133.43,130.89,130.25,128.76,128.56$, 128.20, 127.92, 124.71, 65.01, 46.08, 45.59, 28.52.

IR (KBr): 1716.80, 1697.27, 1605.29, 1520.97, 1347.75, 1173.28, 859.25, $696.48 \mathrm{~cm}^{-1}$ HRMS (ESI): $\mathrm{m} / \mathrm{z}$ calcd for $\mathrm{C}_{20} \mathrm{H}_{18} \mathrm{~N}_{3} \mathrm{O}_{4},[\mathrm{M}+\mathrm{H}]^{+}, 364.1297$; found: 364.1305 .

\section{(Z)-4-(8-benzylidene-1,7-dioxohexahydro-1H-pyrazolo[1,2-a]pyridazin-5-yl)benz onitrile (3j)}

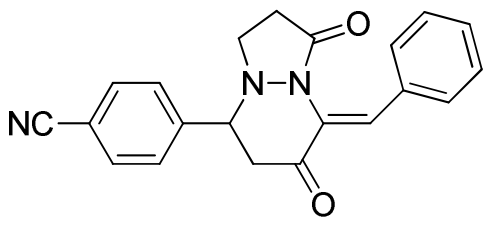

yellow solid (56 mg, $54 \%$ yield); $\mathrm{mp} .: 218-220{ }^{\circ} \mathrm{C}$

${ }^{1} \mathrm{H}$ NMR $\left(400 \mathrm{MHz}, \mathrm{CDCl}_{3}\right) \delta 7.74(\mathrm{~d}, J=8.0 \mathrm{~Hz}, 2 \mathrm{H}), 7.56-7.49$ (m, 4H), $7.48(\mathrm{~s}, 1 \mathrm{H}), 7.37$ (d, $J=$ $6.0 \mathrm{~Hz}, 3 \mathrm{H}), 4.44(\mathrm{dd}, J=10.2,4.7 \mathrm{~Hz}, 1 \mathrm{H}), 3.69-3.49(\mathrm{~m}, 1 \mathrm{H}), 3.22-3.04(\mathrm{~m}, 1 \mathrm{H}), 2.95(\mathrm{dd}, J=$ 18.4, $4.7 \mathrm{~Hz}, 1 \mathrm{H}), 2.91-2.69(\mathrm{~m}, 2 \mathrm{H}), 2.46(\mathrm{dd}, J=17.1,7.8 \mathrm{~Hz}, 1 \mathrm{H})$.

${ }^{13} \mathrm{C}$ NMR $\left(100 \mathrm{MHz}, \mathrm{CDCl}_{3}\right) \delta 190.36,169.63,144.63,133.41,133.28,130.88,130.24,128.76,128.55$, 128.02, 127.90, 118.18, 112.88, 65.32, 46.01, 45.59, 28.53.

IR (KBr): 2228.27, 1716.35, 1696.43, 1606.69, 1393.33, 1172.73, 848.14, 757.28, 733.85, $692.86 \mathrm{~cm}^{-1}$ HRMS (ESI): $\mathrm{m} / \mathrm{z}$ calcd for $\mathrm{C}_{21} \mathrm{H}_{18} \mathrm{~N}_{3} \mathrm{O}_{2}$, [M+H] $]^{+}, 344.1399$; found: 344.1394 .

\section{(Z)-8-benzylidene-5-(4-(trifluoromethyl)phenyl)tetrahydro-1H-pyrazolo[1,2-a]py ridazine-1,7(8H)-dione $(3 \mathrm{k})$}<smiles>O=C1CC(c2ccc(C(F)(F)F)cc2)N2CCC(=O)N2/C1=C\c1ccccc1</smiles>

yellow solid (97 mg, $84 \%$ yield); $\mathrm{mp} .: 83-85^{\circ} \mathrm{C}$

1H NMR (400 MHz, CDCl3) $\delta 7.69(\mathrm{~d}, \mathrm{~J}=8.1 \mathrm{~Hz}, 2 \mathrm{H}), 7.52(\mathrm{~d}, \mathrm{~J}=8.0 \mathrm{~Hz}, 4 \mathrm{H}), 7.48(\mathrm{~s}, 1 \mathrm{H}), 7.40-$ $7.31(\mathrm{~m}, 3 \mathrm{H}), 4.43(\mathrm{dd}, \mathrm{J}=10.3,4.9 \mathrm{~Hz}, 1 \mathrm{H}), 3.58(\mathrm{td}, \mathrm{J}=12.5,8.5 \mathrm{~Hz}, 1 \mathrm{H}), 3.23-3.09(\mathrm{~m}, 1 \mathrm{H}), 2.95$ $(\mathrm{dd}, \mathrm{J}=18.5,4.9 \mathrm{~Hz}, 1 \mathrm{H}), 2.89-2.77(\mathrm{~m}, 2 \mathrm{H}), 2.44(\mathrm{dd}, \mathrm{J}=17.3,7.9 \mathrm{~Hz}, 1 \mathrm{H})$.

13C NMR (100 MHz, CDCl3) $\delta 190.76,169.79,143.45,133.53,131.25,130.92,130.86,130.13$, 128.51, 128.05, 127.67, 126.45, 126.41, 125.15, 122.45, 77.39, 77.07, 76.75, 65.31, 45.84, 28.56.

IR (KBr): 1718.44, 1698.42, 1607.57, 1394.44, 1325.32, 1169.38, 1124.76, 1067.29, 848.97, 692.68 $\mathrm{cm}^{-1}$

HRMS (ESI): $\mathrm{m} / \mathrm{z}$ calcd for $\mathrm{C}_{21} \mathrm{H}_{18} \mathrm{~F}_{3} \mathrm{~N}_{2} \mathrm{O}_{2},[\mathrm{M}+\mathrm{H}]^{+}, 387.1320$; found: 387.1323 .

\section{(Z)-8-benzylidene-5-(2,4-dichlorophenyl)tetrahydro-1H-pyrazolo[1,2-a]pyridazin e-1,7(8H)-dione (3I)}




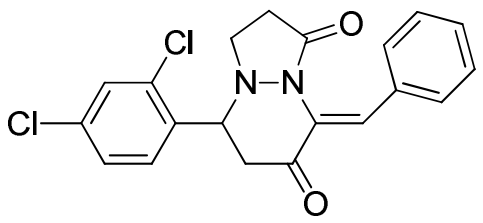

yellow solid (99 mg, $85 \%$ yield); mp.: $205-207^{\circ} \mathrm{C}$

${ }^{1} \mathrm{H}$ NMR $\left(400 \mathrm{MHz}, \mathrm{CDCl}_{3}\right) \delta 7.57-7.50(\mathrm{~m}, 3 \mathrm{H}), 7.48(\mathrm{~s}, 1 \mathrm{H}), 7.46(\mathrm{~d}, J=2.0 \mathrm{~Hz}, 1 \mathrm{H}), 7.38(\mathrm{dd}, J=$ 7.1, $4.0 \mathrm{~Hz}, 4 \mathrm{H}), 4.95(\mathrm{dd}, J=10.4,4.9 \mathrm{~Hz}, 1 \mathrm{H}), 3.58(\mathrm{td}, J=12.5,8.4 \mathrm{~Hz}, 1 \mathrm{H}), 3.14(\mathrm{dd}, J=12.0,9.1$ $\mathrm{Hz}, 1 \mathrm{H}), 2.99$ (dd, $J=18.6,4.9 \mathrm{~Hz}, 1 \mathrm{H}), 2.96-2.85(\mathrm{~m}, 1 \mathrm{H}), 2.73$ (dd, $J=18.5,10.4 \mathrm{~Hz}, 1 \mathrm{H}), 2.48$ (dd, $J=17.4,8.1 \mathrm{~Hz}, 1 \mathrm{H})$.

${ }^{13} \mathrm{C}$ NMR $\left(100 \mathrm{MHz}, \mathrm{CDCl}_{3}\right) \delta 190.66,170.09,135.58,134.92,134.02,133.48,130.90,130.18,129.76$, 129.18, 128.77, 128.62, 128.54, 127.98, 60.36, 46.04, 44.30, 28.94.

IR (KBr): 1718.59, 1698.16, 1607.08, 1391.20, 1195.34, 1172.37, 756.32, $691.20 \mathrm{~cm}^{-1}$ HRMS (ESI): $\mathrm{m} / \mathrm{z}$ calcd for $\mathrm{C}_{20} \mathrm{H}_{17} \mathrm{Cl}_{2} \mathrm{~N}_{2} \mathrm{O}_{2},[\mathrm{M}+\mathrm{H}]^{+}, 387.0667$; found: 387.0670 .

\section{(Z)-8-benzylidene-5-(naphthalen-2-yl)tetrahydro-1H-pyrazolo[1,2-a]pyridazine-1} ,7(8H)-dione (3m)

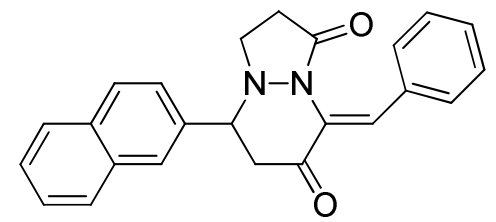

yellow solid (73 mg, $66 \%$ yield); $\mathrm{mp} .: 160-162{ }^{\circ} \mathrm{C}$

${ }^{1} \mathrm{H}$ NMR (400 MHz, $\left.\mathrm{CDCl}_{3}\right) \delta 7.93(\mathrm{~d}, J=8.5 \mathrm{~Hz}, 1 \mathrm{H}), 7.87$ (s, 2H), $7.81(\mathrm{~s}, 1 \mathrm{H}), 7.54(\mathrm{~d}, J=4.6 \mathrm{~Hz}$, $5 \mathrm{H}), 7.50(\mathrm{~s}, 1 \mathrm{H}), 7.36(\mathrm{~d}, J=7.5 \mathrm{~Hz}, 3 \mathrm{H}), 4.59-4.45(\mathrm{~m}, 1 \mathrm{H}), 3.63-3.48(\mathrm{~m}, 1 \mathrm{H}), 3.34-3.20(\mathrm{~m}$, $1 \mathrm{H}), 3.10-2.95(\mathrm{~m}, 2 \mathrm{H}), 2.88(\mathrm{~s}, 1 \mathrm{H}), 2.43(\mathrm{~s}, 1 \mathrm{H})$.

${ }^{13} \mathrm{C}$ NMR $\left(100 \mathrm{MHz}, \mathrm{CDCl}_{3}\right) \delta 191.50,170.06,136.70,133.68,133.45,133.33,130.88,130.02,129.59$, $128.49,128.28,127.94,127.88,126.90,126.83,126.73,124.21,66.02,45.98,45.65,28.75$.

IR (KBr): 1714.83, 1696.64, 1605.38, 1392.93, 1323.22, 1236.27, 1191.20, 1179.51, 752.54, 734.51, $692.27 \mathrm{~cm}^{-1}$

HRMS (ESI): $\mathrm{m} / \mathrm{z}$ calcd for $\mathrm{C}_{24} \mathrm{H}_{21} \mathrm{~N}_{2} \mathrm{O}_{2}$, [M+H] $]^{+}, 369.1603$; found: 369.1609 .

\section{(Z)-5-(4-bromophenyl)-8-(4-methylbenzylidene)tetrahydro-1H-pyrazolo[1,2-a]py ridazine-1,7(8H)-dione (3n)}<smiles>CC1C(=O)/C(=C/c2ccccc2)N2C(=O)CCN2C1c1ccc(Br)cc1</smiles>

yellow solid (101 mg, $82 \%$ yield); $\mathrm{mp} .: 177-179^{\circ} \mathrm{C}$

${ }^{1} \mathrm{H}$ NMR $\left(400 \mathrm{MHz}, \mathrm{CDCl}_{3}\right) \delta 7.57(\mathrm{~d}, J=8.2 \mathrm{~Hz}, 2 \mathrm{H}), 7.53-7.46(\mathrm{~m}, 3 \mathrm{H}), 7.35(\mathrm{~d}, J=7.4 \mathrm{~Hz}, 3 \mathrm{H})$, $7.27(\mathrm{~s}, 1 \mathrm{H}), 7.25(\mathrm{~s}, 1 \mathrm{H}), 3.91(\mathrm{~d}, J=10.0 \mathrm{~Hz}, 1 \mathrm{H}), 3.50(\mathrm{td}, J=12.6,8.6 \mathrm{~Hz}, 1 \mathrm{H}), 3.16-3.05(\mathrm{~m}$, 1H), $2.89-2.68$ (m, 2H), 2.42 (dd, $J=17.5,8.0 \mathrm{~Hz}, 1 \mathrm{H}), 1.05$ (d, $J=7.0 \mathrm{~Hz}, 3 \mathrm{H})$. 
${ }^{13} \mathrm{C}$ NMR $\left(100 \mathrm{MHz}, \mathrm{CDCl}_{3}\right) \delta 193.97,169.83,137.87,133.78,132.50,130.74,129.93,129.48,128.47$, $128.32,127.71,122.85,72.24,49.38,45.79,28.49,12.93$.

IR (KBr): 1717.61, 1694.72, 1607.82, 1448.96, 1394.33, 1191.54, 1173.72, 1073.16, 1009.81, 735.31, $691.87 \mathrm{~cm}^{-1}$

HRMS (ESI): $\mathrm{m} / \mathrm{z}$ calcd for $\mathrm{C}_{21} \mathrm{H}_{20} \mathrm{BrN}_{2} \mathrm{O}_{2},[\mathrm{M}+\mathrm{H}]^{+}, 411.0708$; found: 411.0705 .

\section{(Z)-8-benzylidene-5-(4-bromophenyl)-6-methyltetrahydro-1H-pyrazolo[1,2-a]pyr} idazine-1,7(8H)-dione (3o)

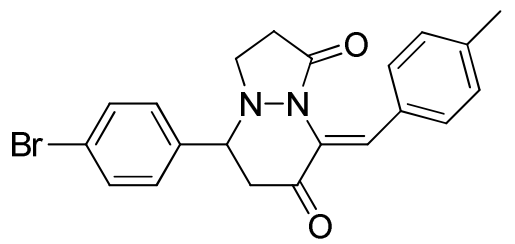

yellow solid ( $82 \mathrm{mg}, 66 \%$ yield); $\mathrm{mp} .: 186-188^{\circ} \mathrm{C}$

${ }^{1} \mathrm{H}$ NMR $\left(400 \mathrm{MHz}, \mathrm{CDCl}_{3}\right) \delta 7.56(\mathrm{~d}, J=8.3 \mathrm{~Hz}, 2 \mathrm{H}), 7.46(\mathrm{~s}, 1 \mathrm{H}), 7.41(\mathrm{~d}, J=8.1 \mathrm{~Hz}, 2 \mathrm{H}), 7.28(\mathrm{~s}$, $1 \mathrm{H}), 7.25(\mathrm{~s}, 1 \mathrm{H}), 7.17(\mathrm{~d}, J=8.0 \mathrm{~Hz}, 2 \mathrm{H}), 4.31(\mathrm{dd}, J=10.1,5.0 \mathrm{~Hz}, 1 \mathrm{H}), 3.55(\mathrm{td}, J=12.4,8.6 \mathrm{~Hz}$, 1H), $3.26-3.14$ (m, 1H), 2.92 (dd, $J=18.5,4.9 \mathrm{~Hz}, 1 \mathrm{H}), 2.82$ (dd, $J=18.5,10.2 \mathrm{~Hz}, 2 \mathrm{H}), 2.44$ (s, 1H), $2.36(\mathrm{~s}, 3 \mathrm{H})$.

${ }^{13} \mathrm{C}$ NMR $\left(100 \mathrm{MHz}, \mathrm{CDCl}_{3}\right) \delta 191.04,169.85,140.67,138.53,132.58,131.04,130.71,129.31,128.96$, 128.64, 127.47, 122.77, 65.21, 45.88, 45.68, 28.67, 21.69.

IR (KBr): $1715.68,1697.96,1598.44,1392.41,1172.43,1011.05,896.50,827.95,813.49 \mathrm{~cm}^{-1}$ HRMS (ESI): $\mathrm{m} / \mathrm{z}$ calcd for $\mathrm{C}_{21} \mathrm{H}_{20} \mathrm{BrN}_{2} \mathrm{O}_{2},[\mathrm{M}+\mathrm{H}]^{+}, 411.0708$; found: 411.0709 .

\section{(Z)-5-(4-bromophenyl)-8-(4-fluorobenzylidene)tetrahydro-1H-pyrazolo[1,2-a]pyr idazine-1,7(8H)-dione (3p)}

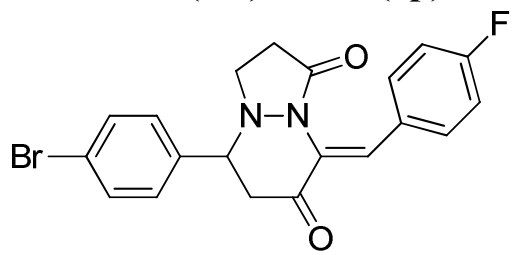

yellow solid ( $80 \mathrm{mg}, 64 \%$ yield); $\mathrm{mp} .: 191-193{ }^{\circ} \mathrm{C}$

${ }^{1} \mathrm{H}$ NMR (400 MHz, $\mathrm{CDCl}_{3}$ ) $\delta 7.57$ (d, $\left.J=8.3 \mathrm{~Hz}, 2 \mathrm{H}\right), 7.50(\mathrm{dd}, J=8.4,5.6 \mathrm{~Hz}, 2 \mathrm{H}), 7.43(\mathrm{~s}, 1 \mathrm{H})$, 7.27 (s, $1 \mathrm{H}), 7.25(\mathrm{~s}, 1 \mathrm{H}), 7.06$ (t, $J=8.6 \mathrm{~Hz}, 2 \mathrm{H}), 4.32(\mathrm{dd}, J=10.1,4.8 \mathrm{~Hz}, 1 \mathrm{H}), 3.54(\mathrm{td}, J=12.3$, $8.5 \mathrm{~Hz}, 1 \mathrm{H}), 3.30-3.17(\mathrm{~m}, 1 \mathrm{H}), 2.93(\mathrm{dd}, J=18.5,4.7 \mathrm{~Hz}, 1 \mathrm{H}), 2.88-2.75(\mathrm{~m}, 2 \mathrm{H}), 2.44(\mathrm{~s}, 1 \mathrm{H})$.

${ }^{13} \mathrm{C} \mathrm{NMR}\left(100 \mathrm{MHz}, \mathrm{CDCl}_{3}\right) \delta 190.93,169.98,164.56,162.05,138.36,133.00,132.91,132.63,129.81$, $128.92,127.77,127.10,122.87,115.84,115.63,65.15,45.85,45.69,28.62$.

IR (KBr): 1715.71, 1596.79, 1507.36, 1391.76, 1226.11, 1172.08, 1159.16, 1011.37, 829.94, 736.34 $\mathrm{cm}^{-1}$

HRMS (ESI): $\mathrm{m} / \mathrm{z}$ calcd for $\mathrm{C}_{20} \mathrm{H}_{17} \mathrm{BrFN}_{2} \mathrm{O}_{2}$, [M+H] $]^{+}, 415.0457$; found: 415.0450 . 
NMR spectra for all new compounds

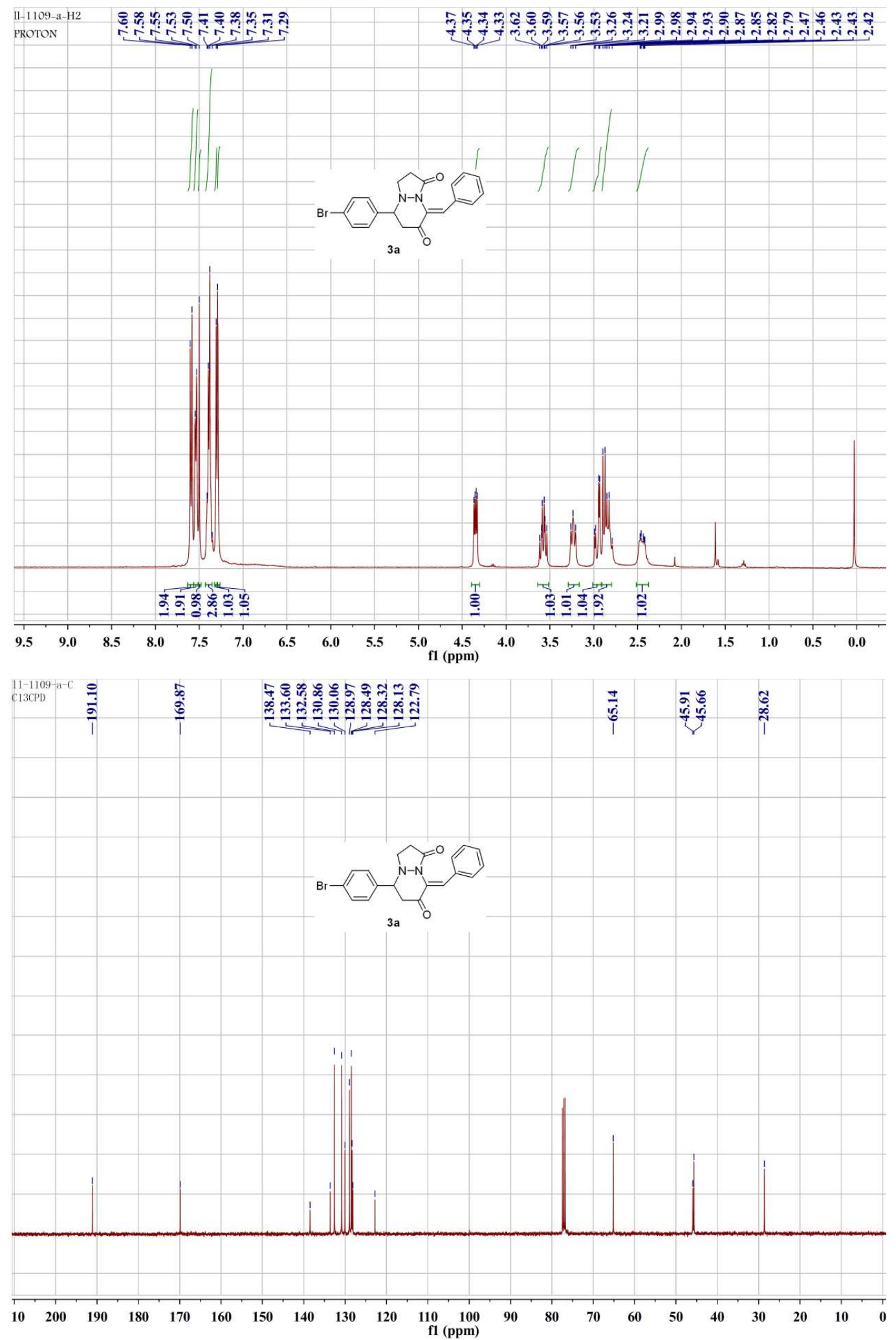



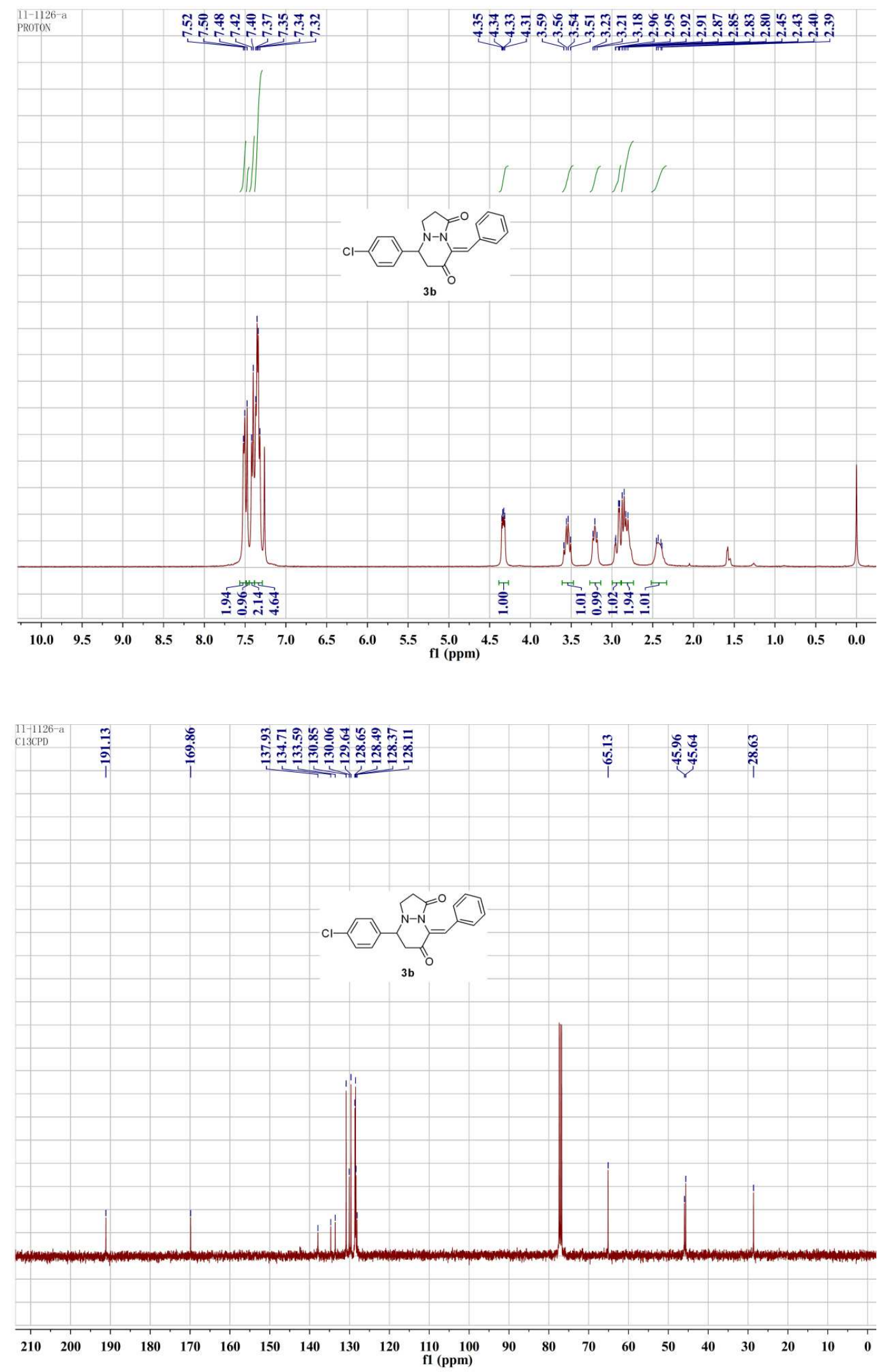

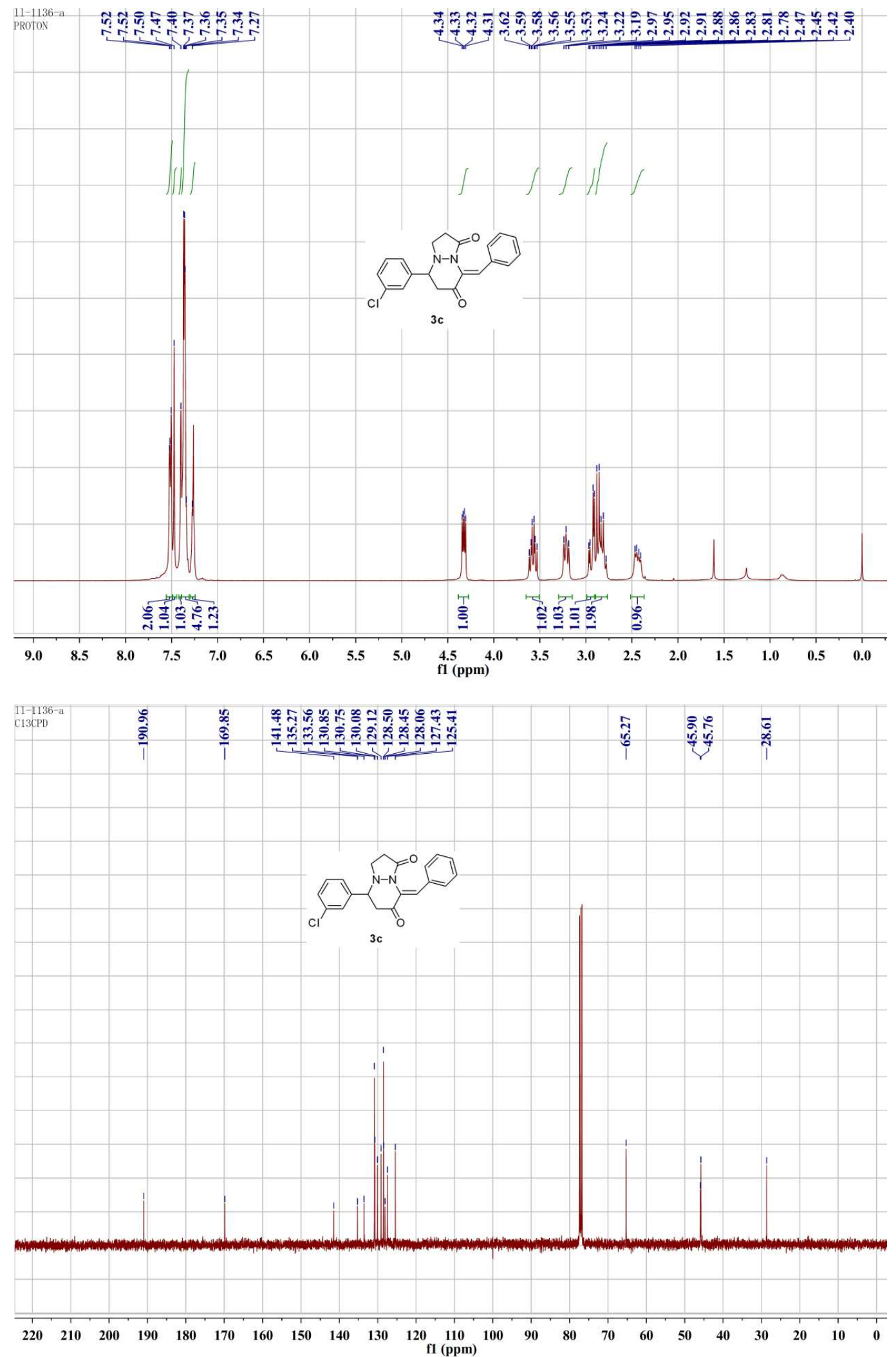

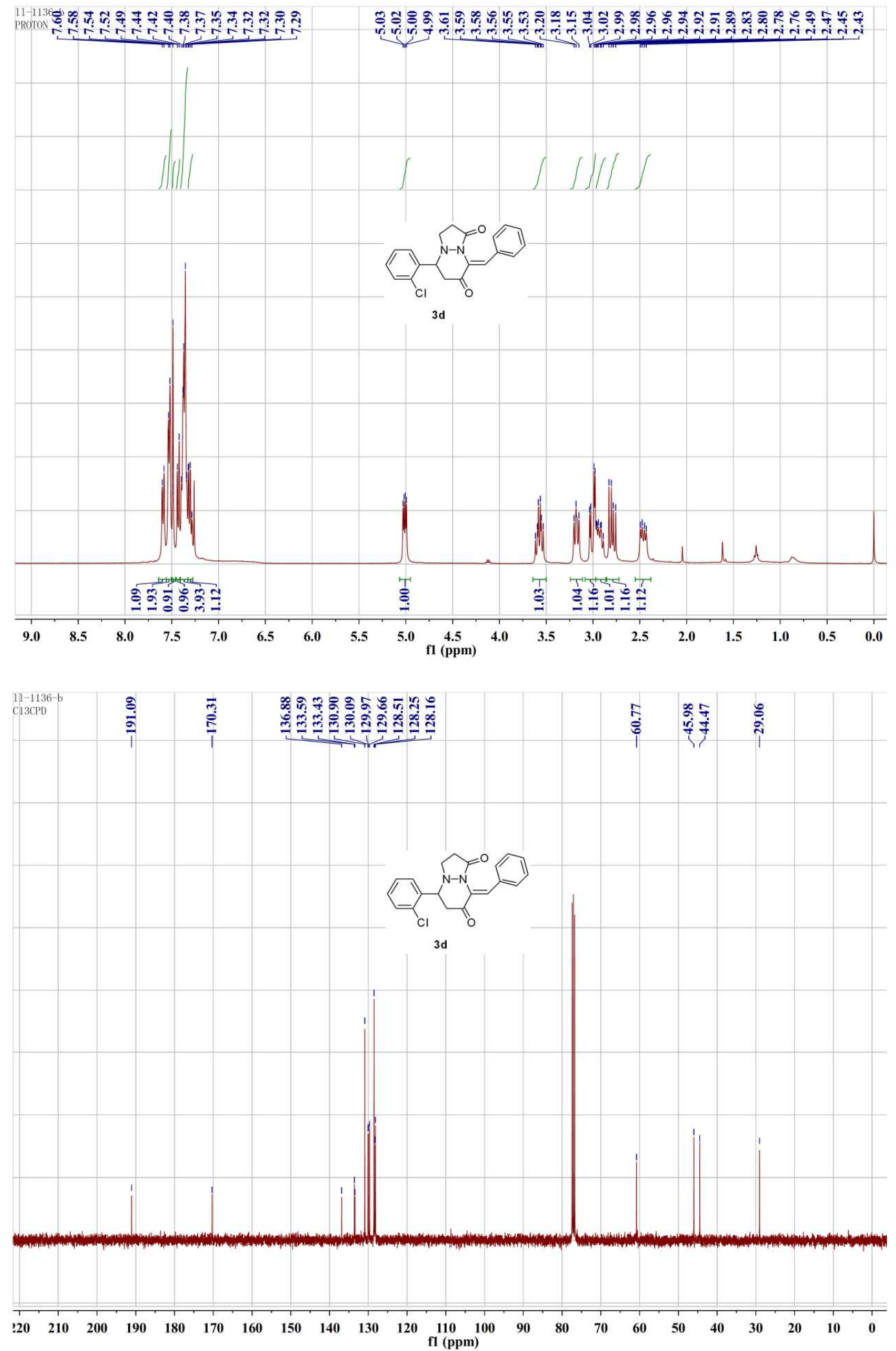

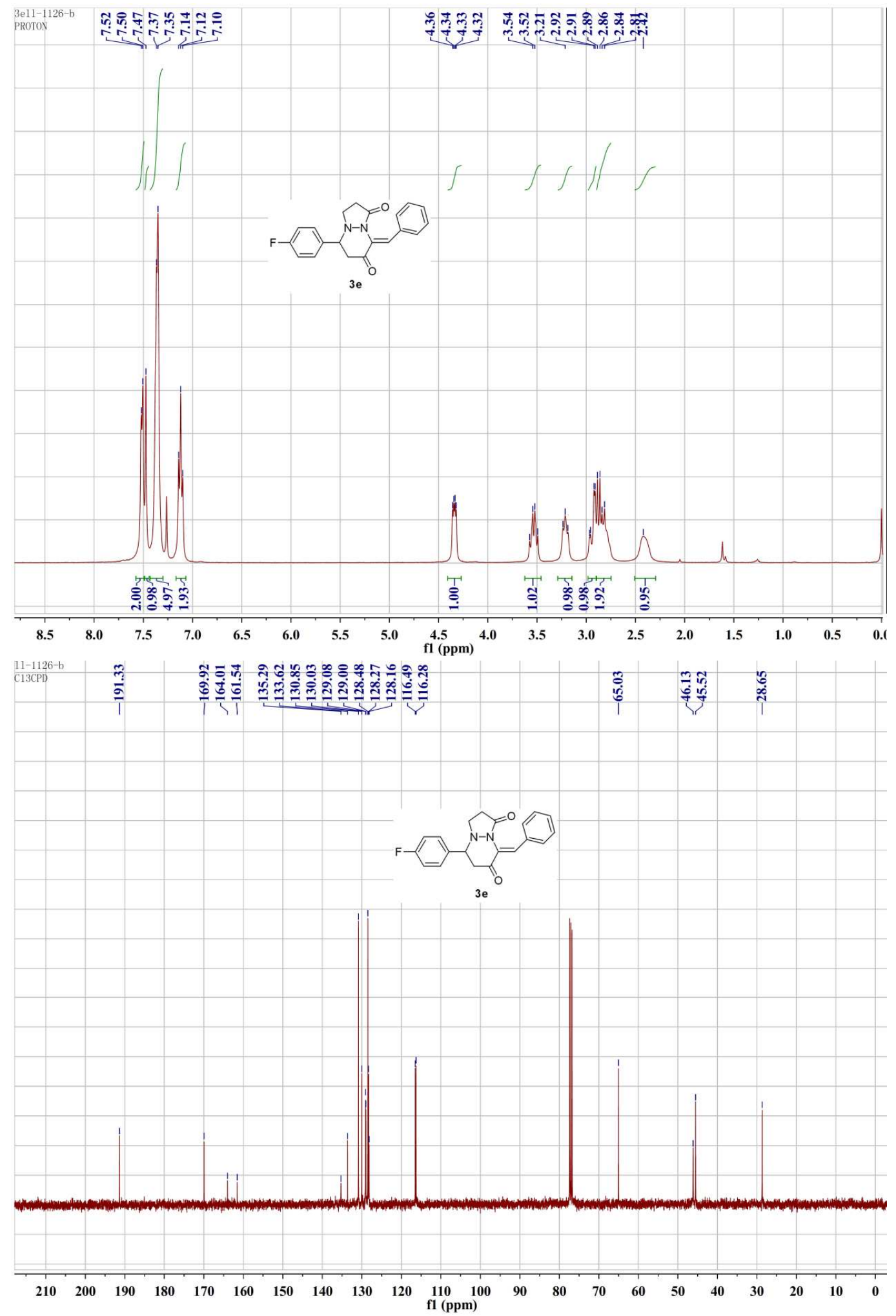

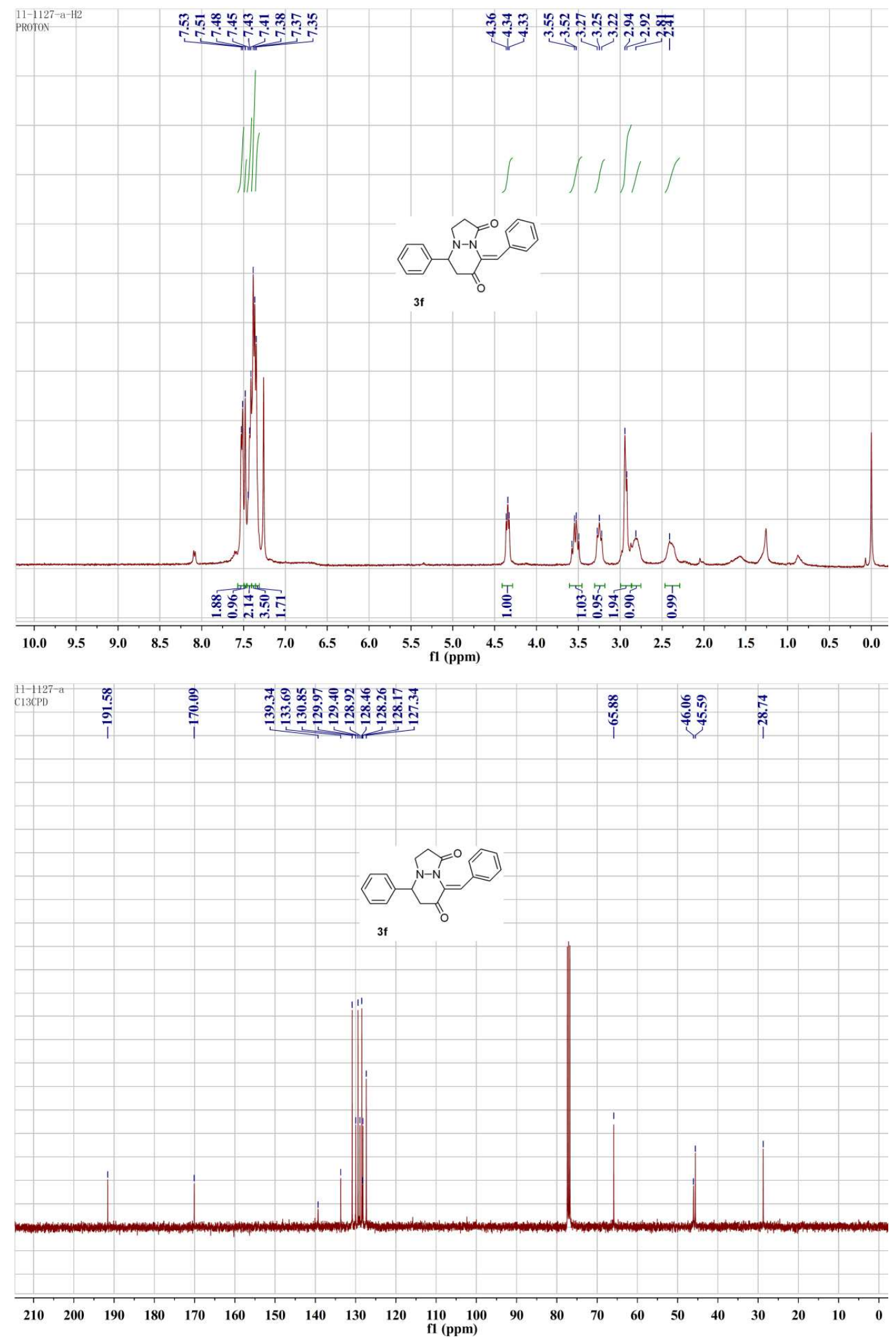

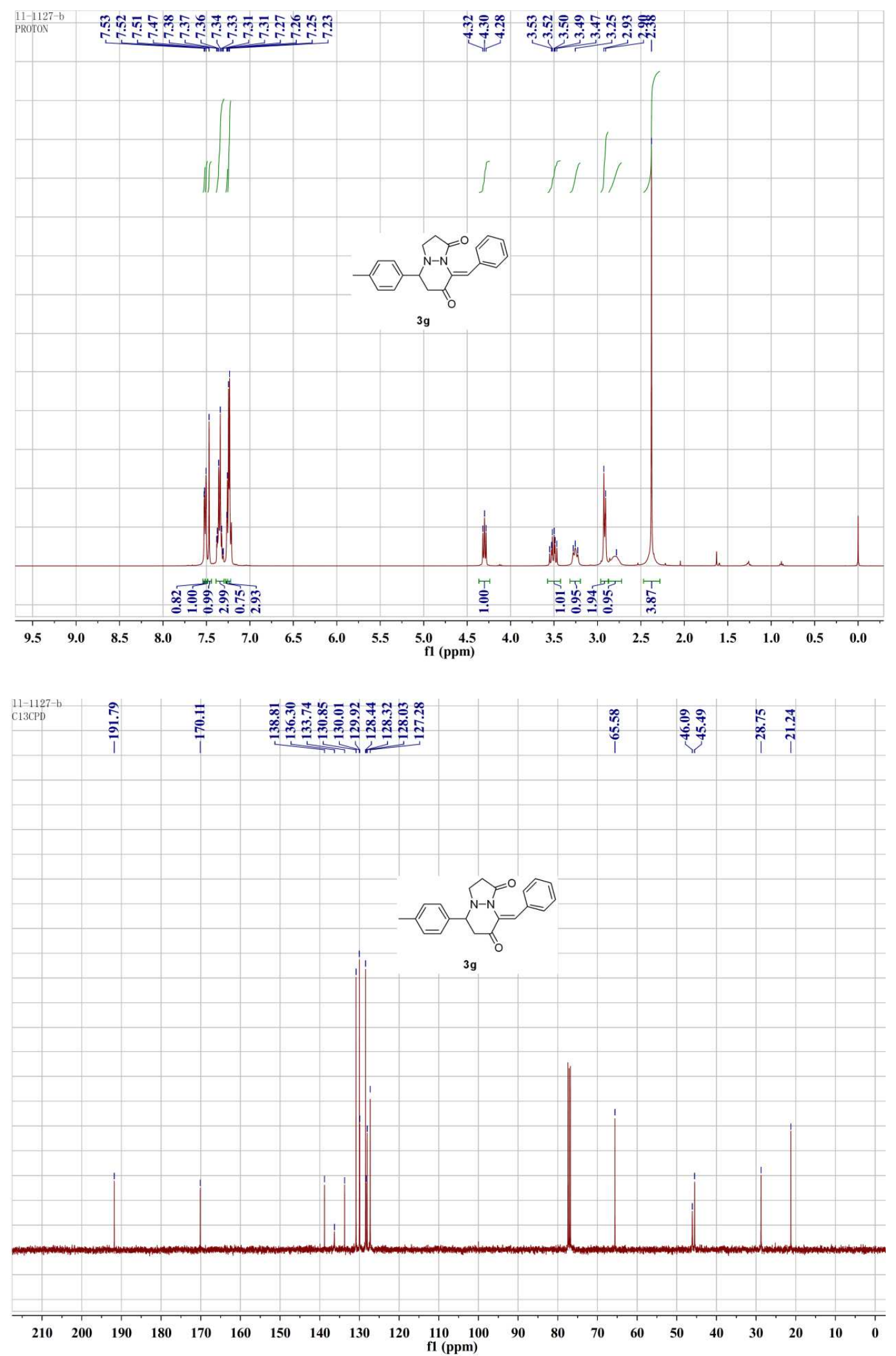

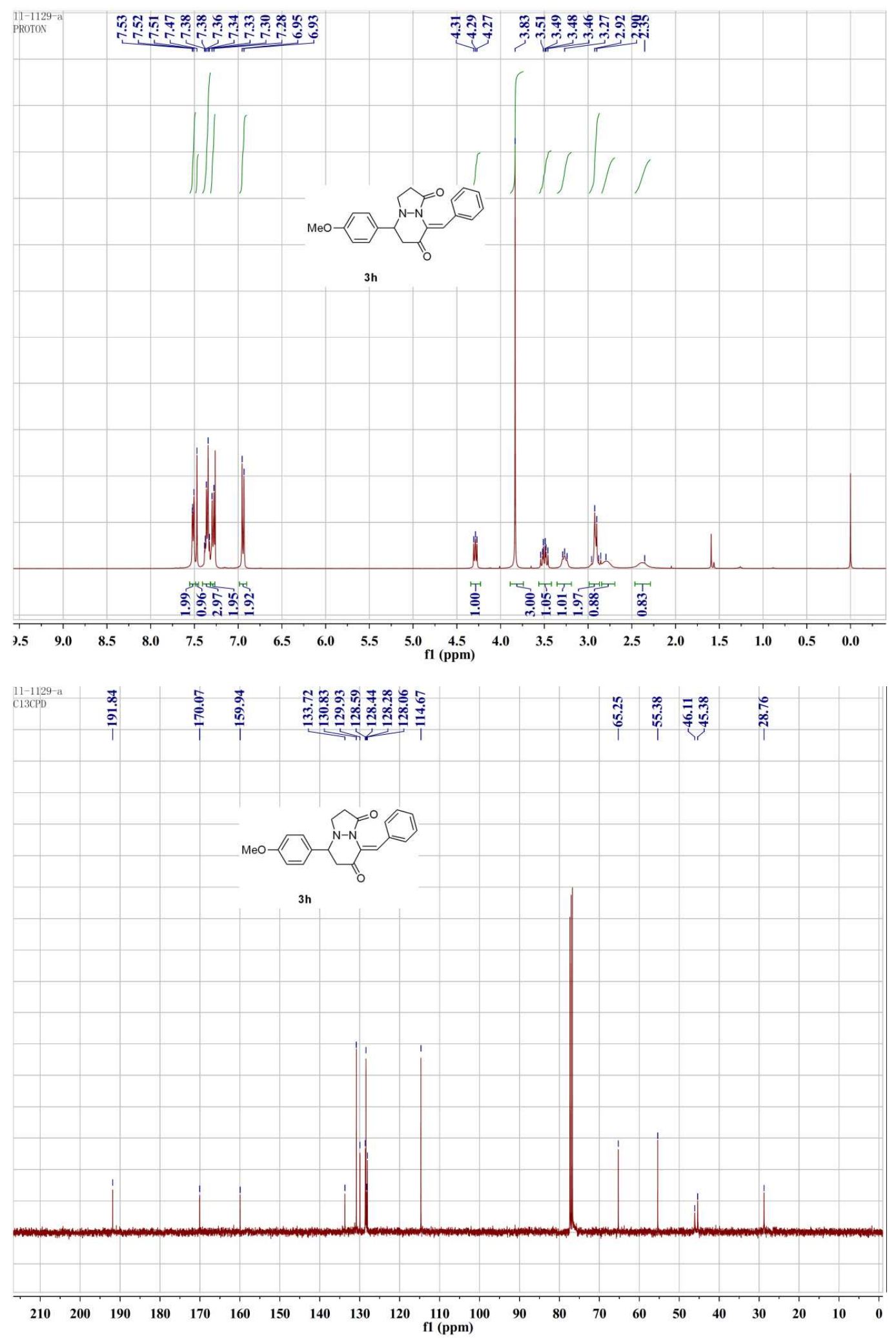

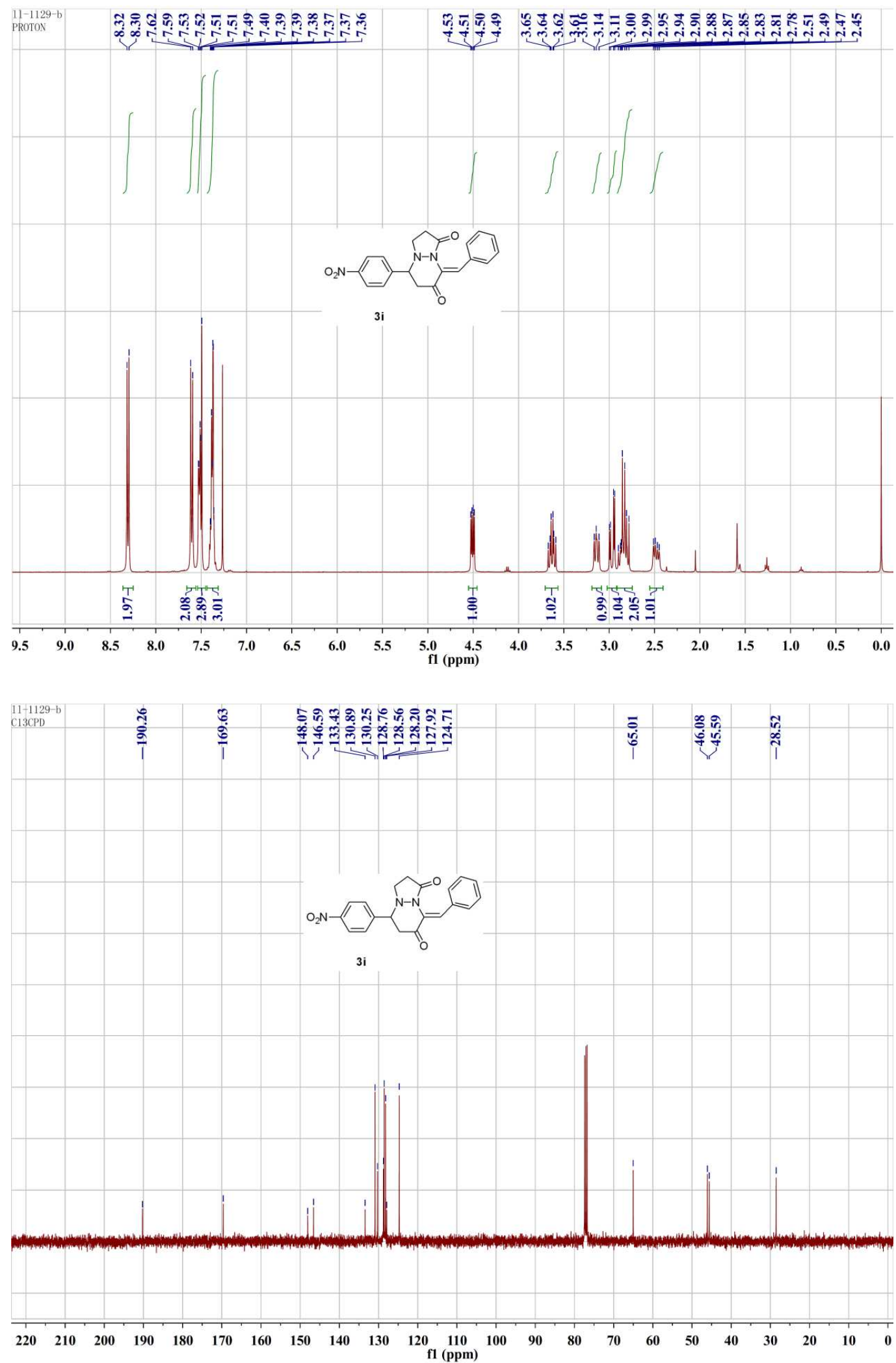


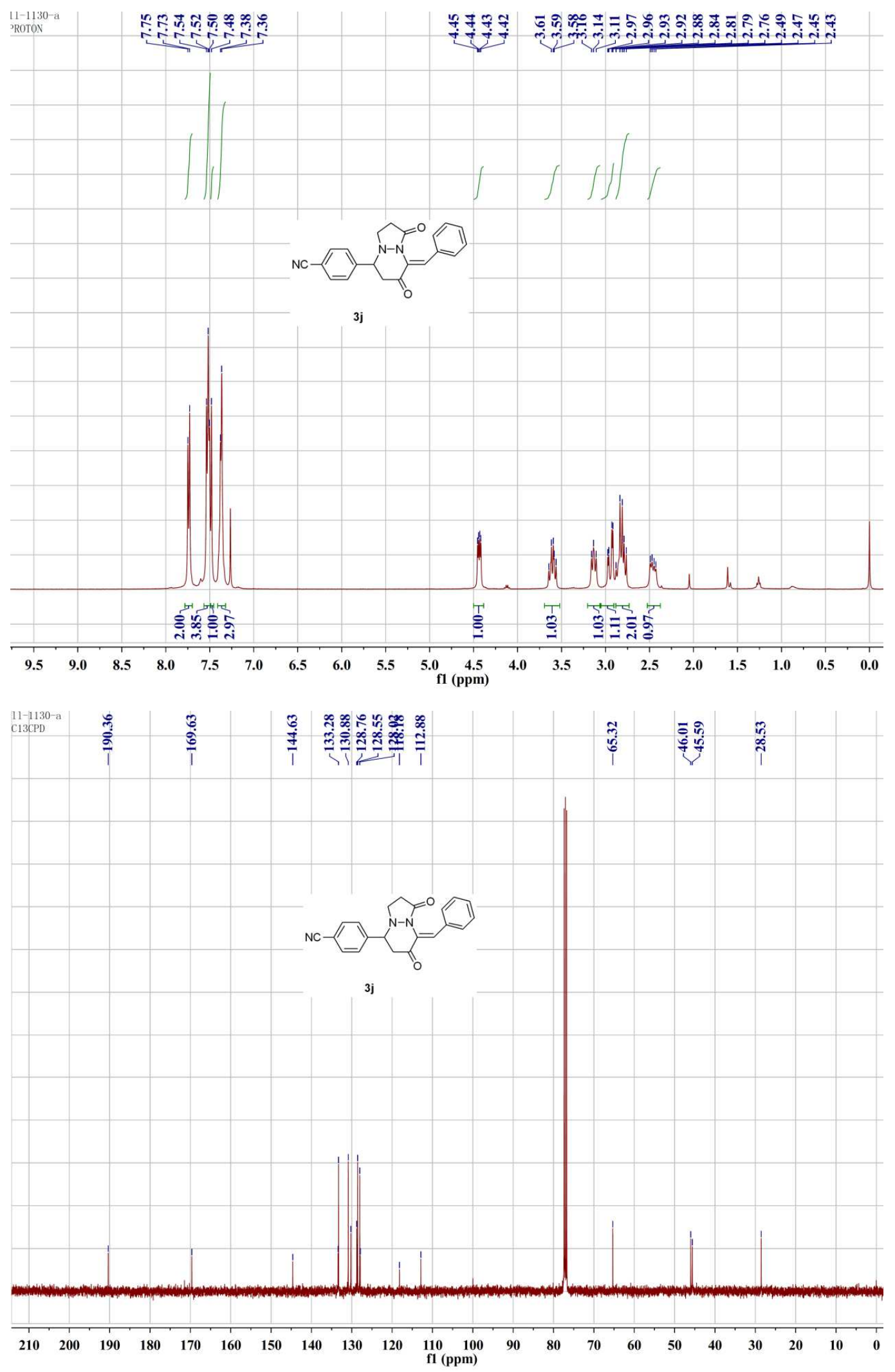



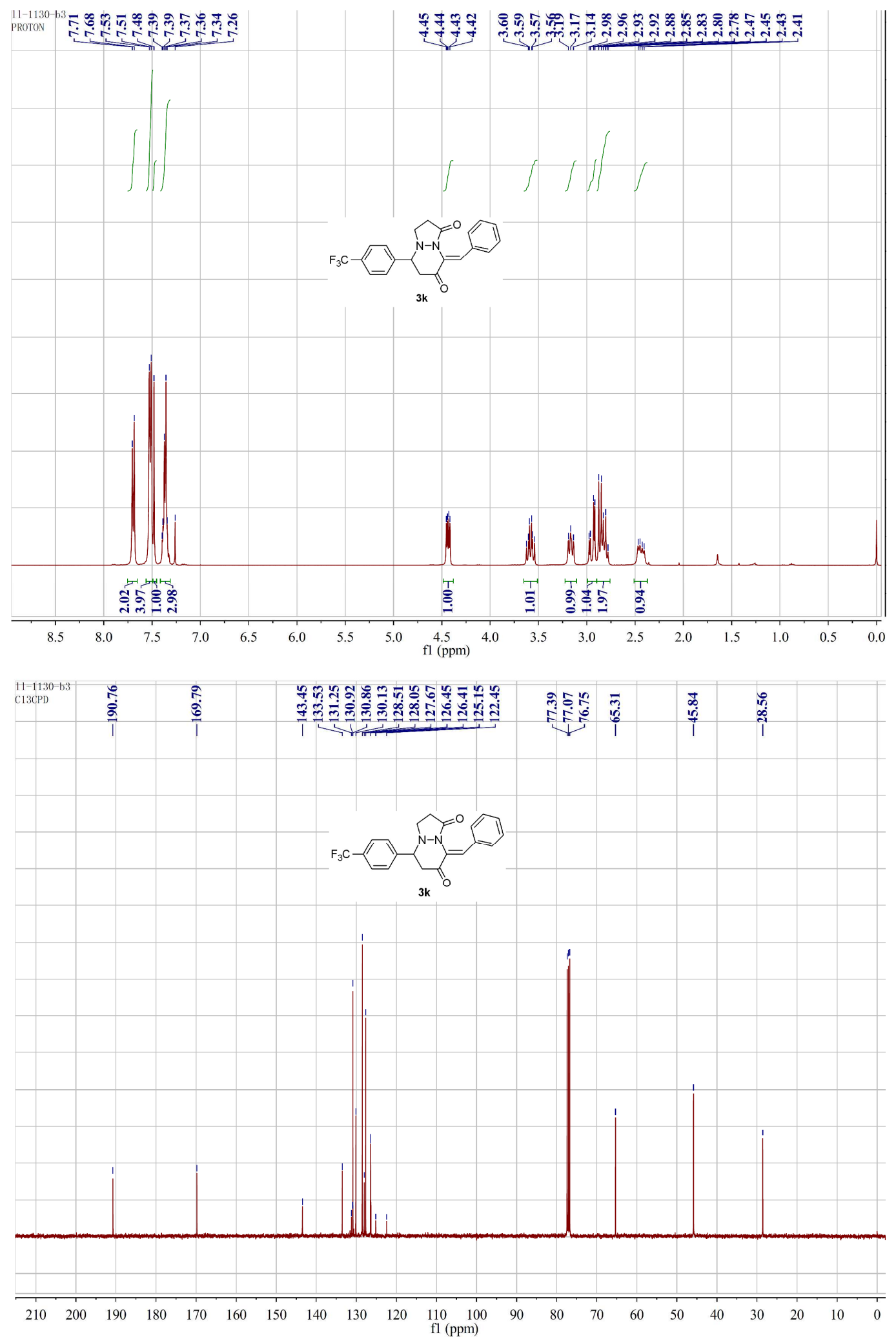

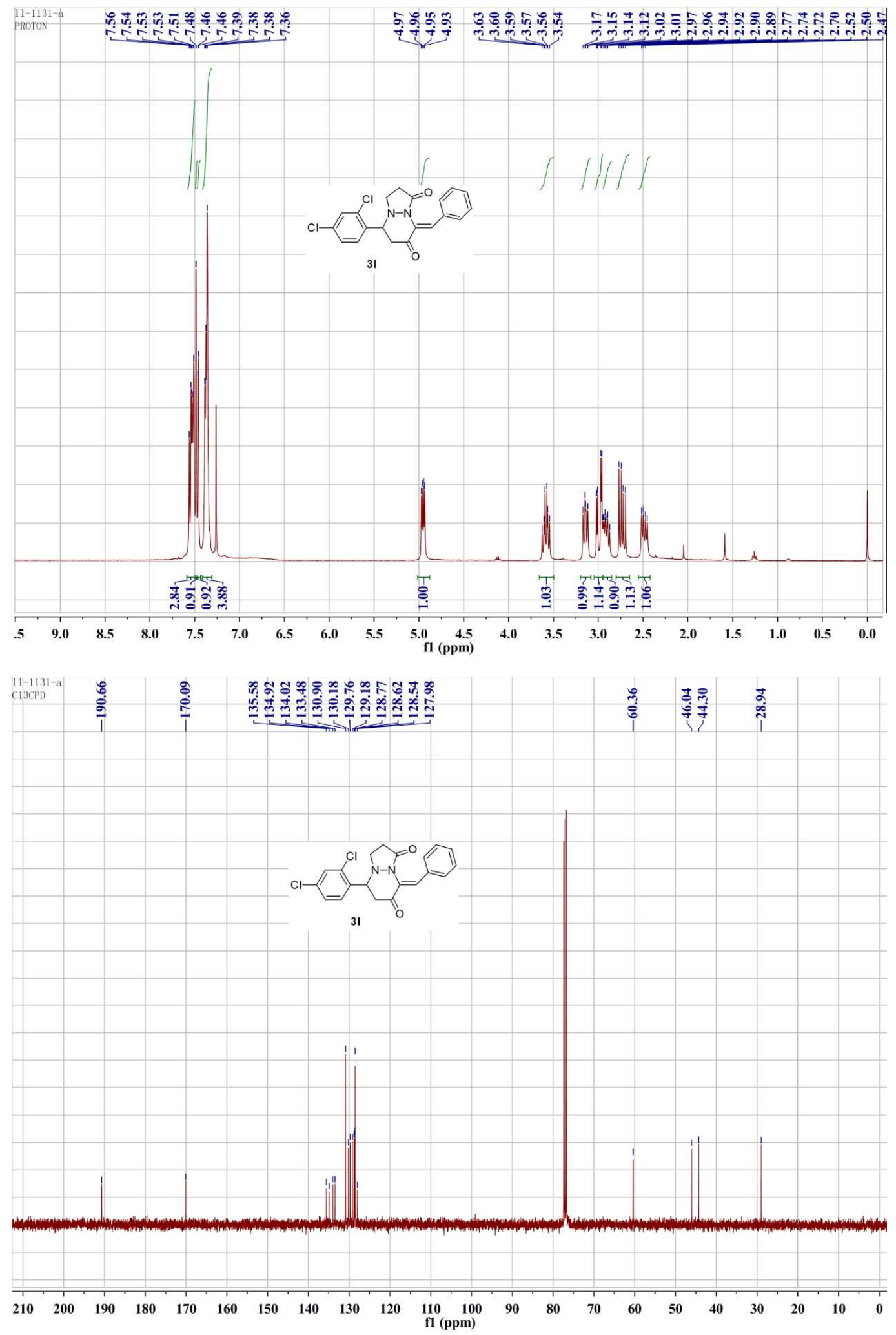

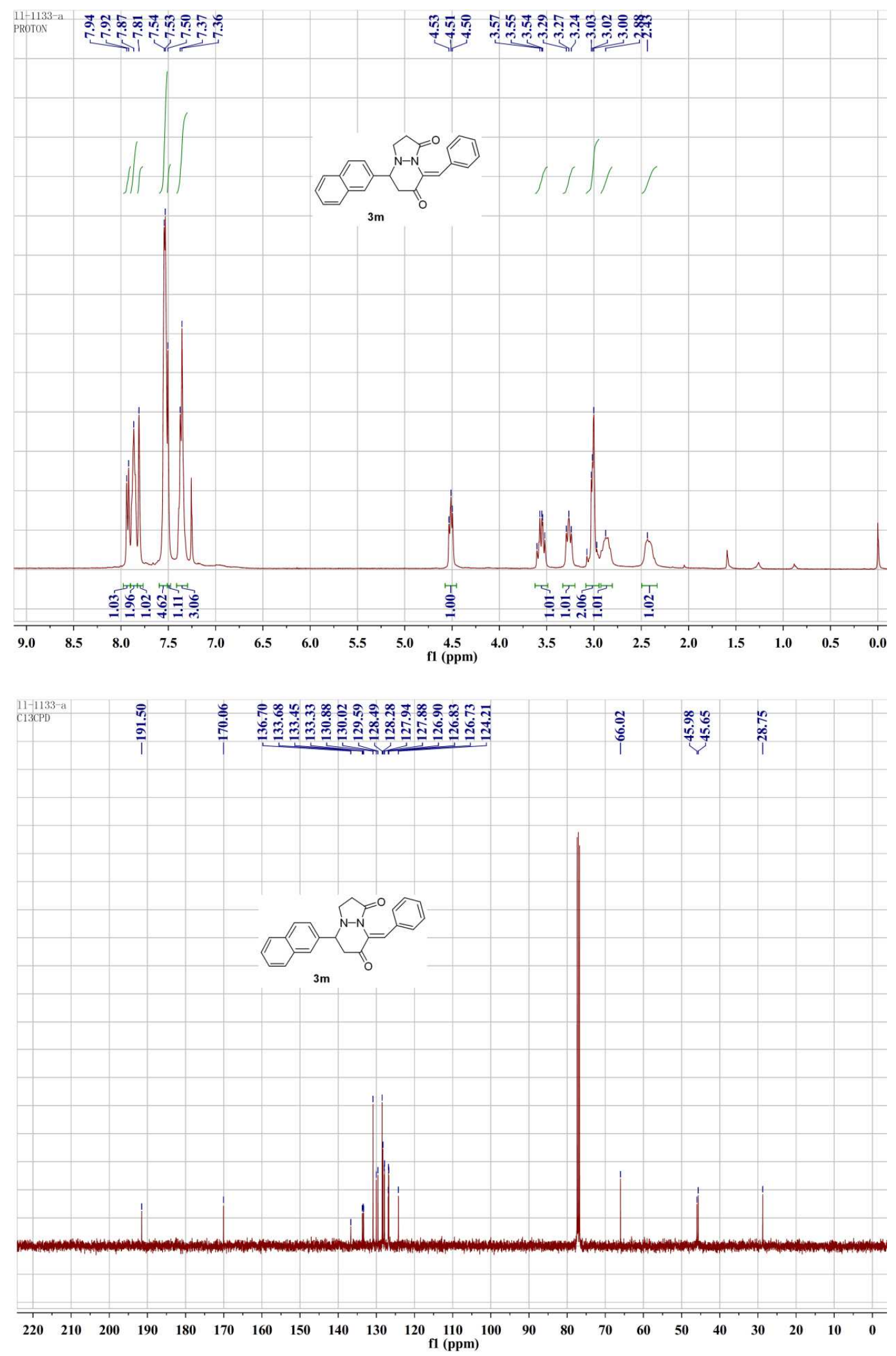

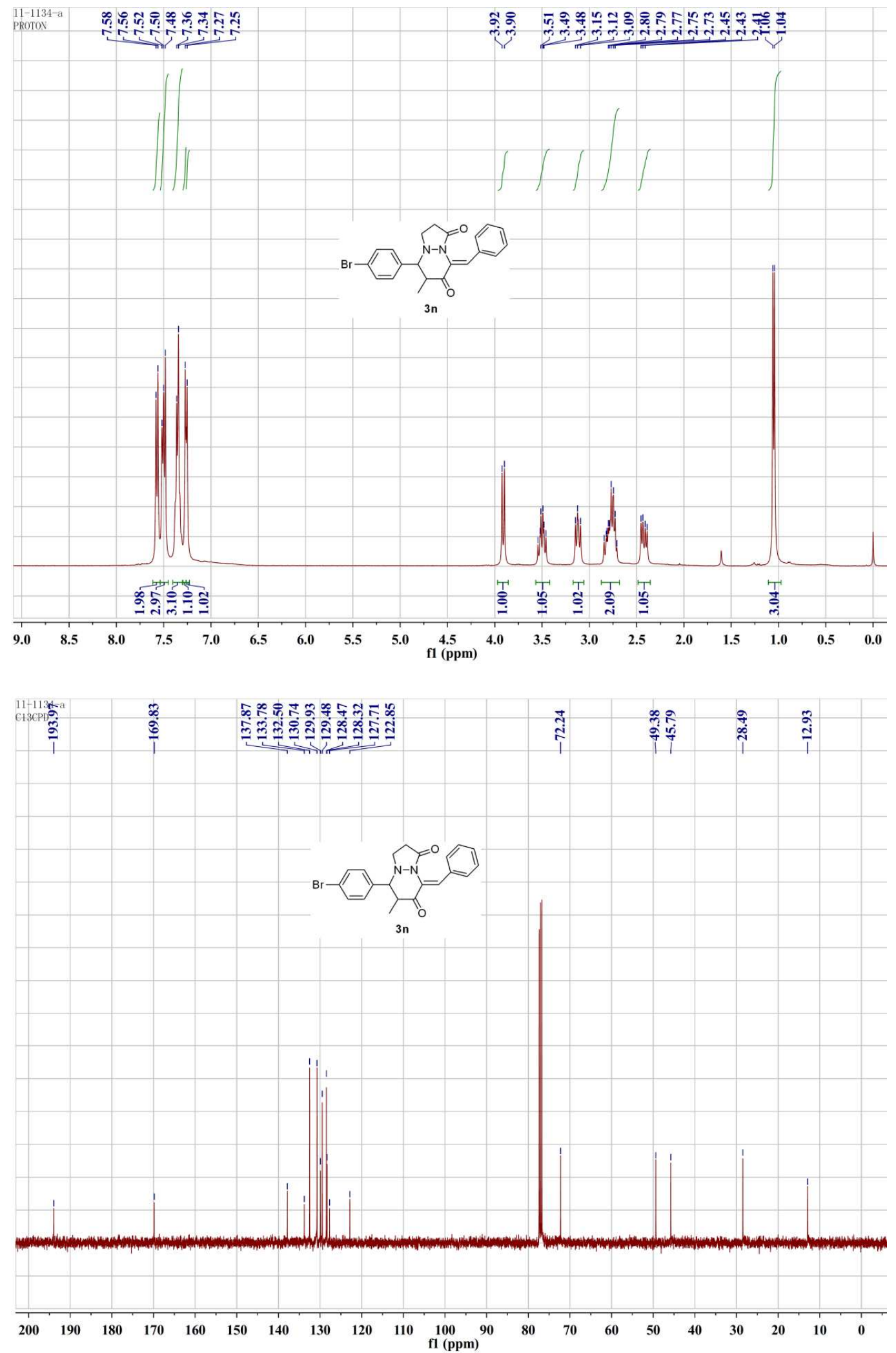

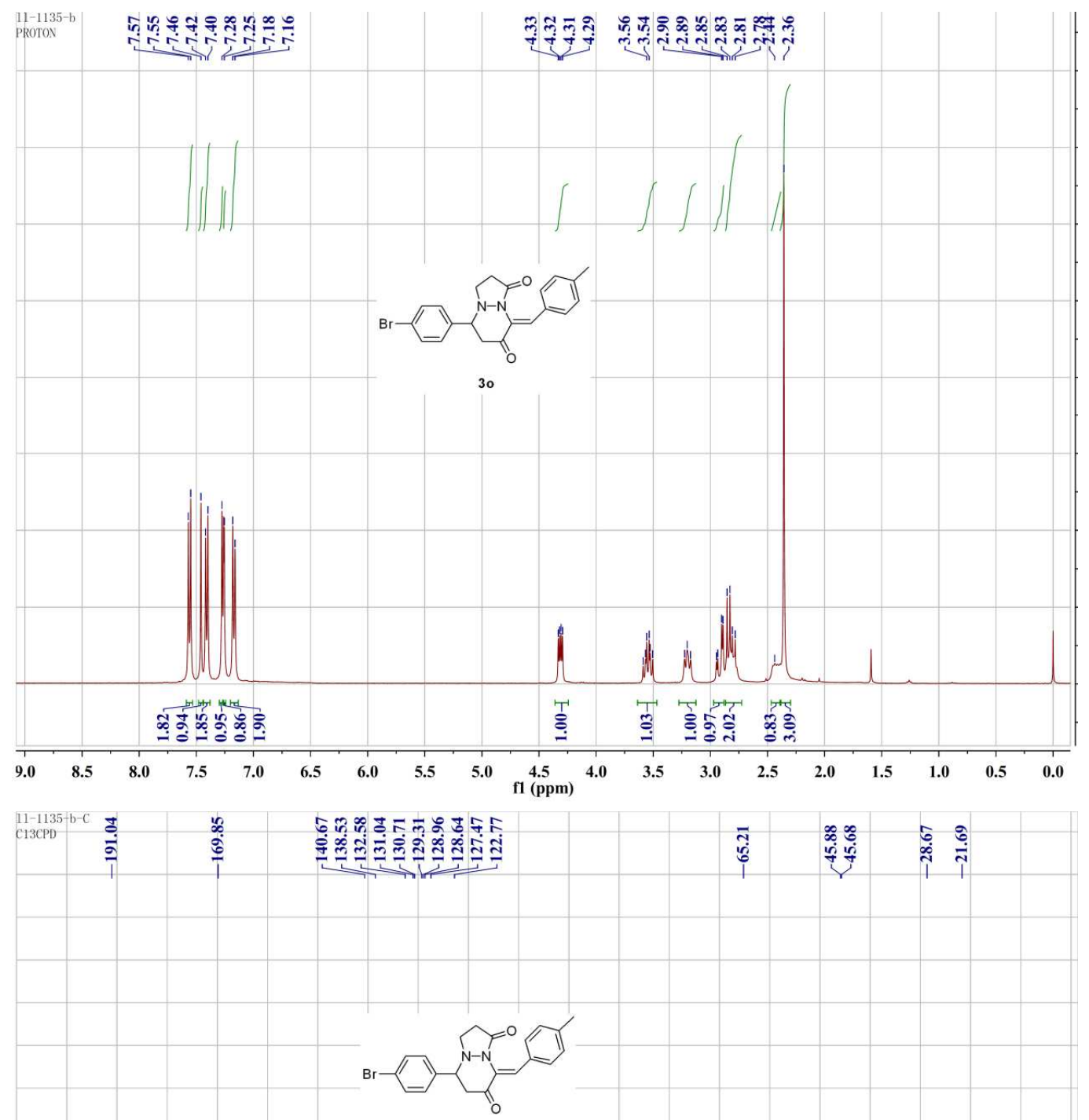

3o
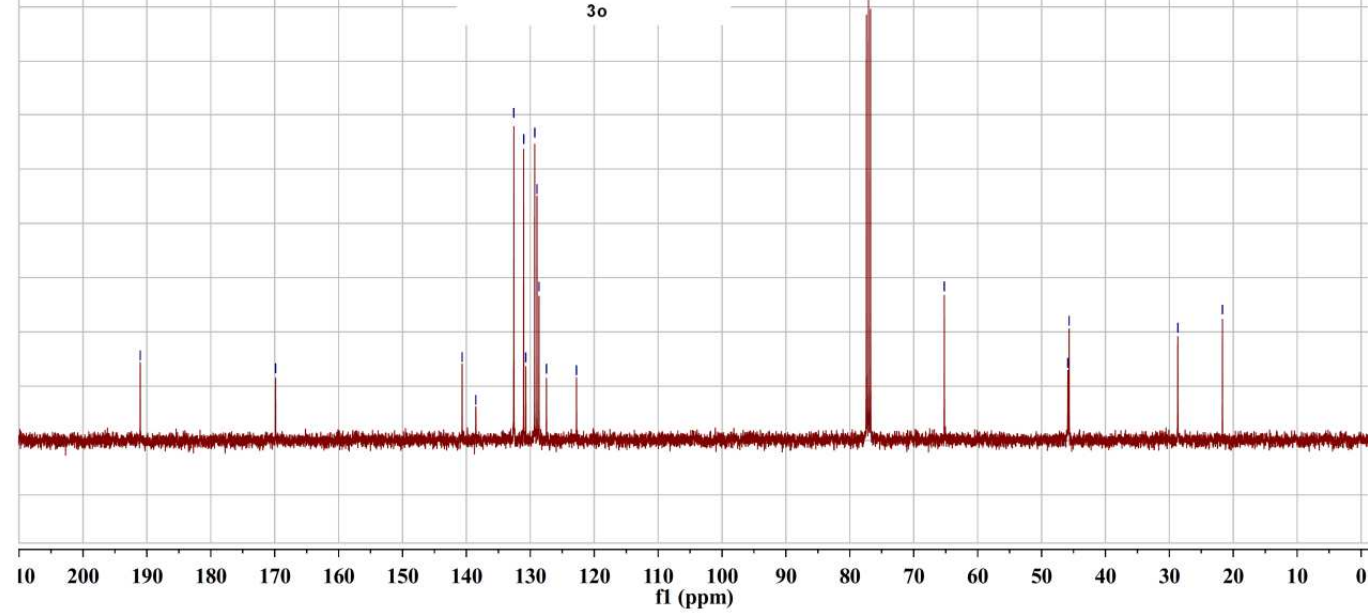

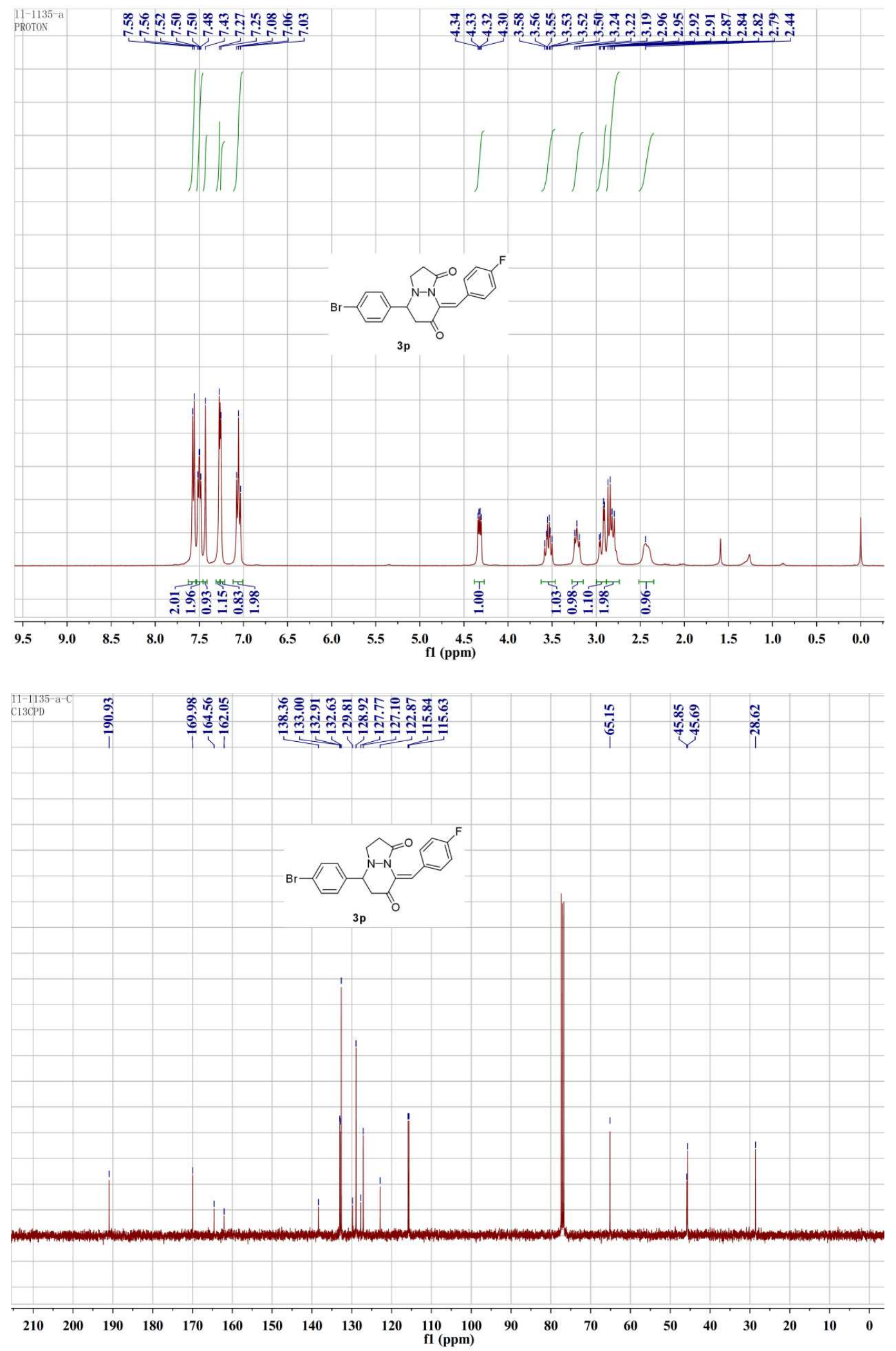


\section{X-ray crystal structure of $3 \mathrm{a}$}

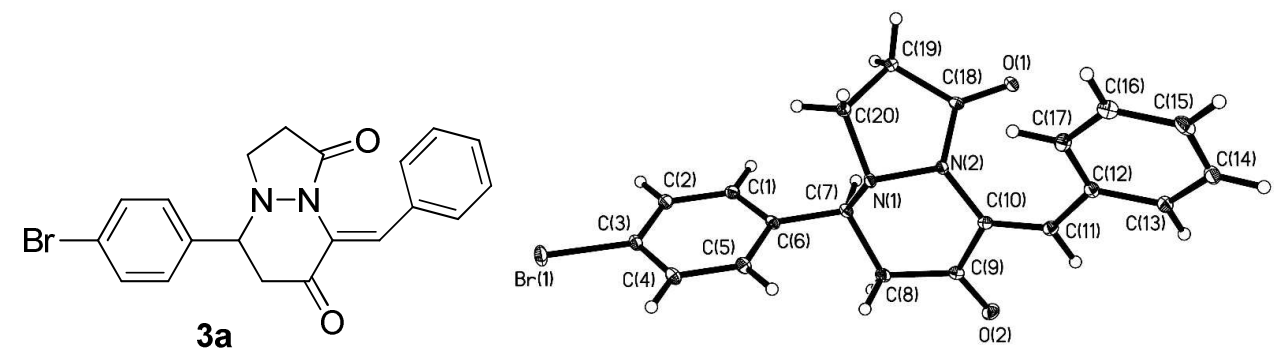

S-Figure 1. X-ray structure of $\mathbf{3 a}$ 